\title{
Dielectric and Insulating Properties of an Acrylic DEA Material at High near-DC Electric Fields
}

\author{
L. Di Lillo ${ }^{a}$, A. Schmidt ${ }^{b} c$, A. Bergamini ${ }^{c}$, P. Ermanni $^{a}$, E. Mazza $^{b} c$ \\ ${ }^{a}$ ETH Zurich, Centre for Structure Technologies, Leonhardstrasse 27, 8092 Zurich, Switzerland; \\ ${ }^{b}$ ETH Zurich, Institute for Mechanical Systems, Tannenstrasse 3, 8092 Zurich, Switzerland; \\ ${ }^{c}$ Empa, Materials Science and Technology, berlandstrasse 129, 8600 Dübendorf, Switzerland;
}

\begin{abstract}
A number of adaptive structure applications call for the generation of intense electric fields (in excess of $70 \mathrm{MV} / \mathrm{m}$ ). Such intense fields across the thickness of a thin polymer dielectric layer are typically used to exploit the direct electromechanical coupling in the form of a Maxwell stress:

$$
\sigma_{i j}=-\frac{1}{2} \cdot \epsilon \cdot \epsilon_{0} \cdot \frac{V^{2}}{d^{2}}\left[\begin{array}{ccc}
1 & 0 & 0 \\
0 & 1 & 0 \\
0 & 0 & -1
\end{array}\right]
$$

Where V/d is the applied field, $\epsilon_{0}$ is the permittivity of vacuum and $\epsilon$ is the relative permittivity of the material. The field that can be applied to the dielectric is limited by the dielectric strength of the material. Below the limit set by the breakdown, the material is generally assumed to have a field independent dielectric constant and to be a perfect insulator, i.e. to have an infinite volume resistivity. While extensive investigations about the mechanical properties of the materials used for electronic Dielectric Elastomer Actuators (DEA) are available from literature, the results of the investigation of the insulating and dielectric properties of these materials, especially under conditions (electric field and frequency) similar to the ones encountered during operation are not available. In the present contribution, we present a method and a set-up for the measurement of the electric properties of thin polymer films, such as the ones used for the fabrication of electronic DEAs, under conditions close to operations. The method and setup where developed to investigate the properties of 'stiff' thin polymer films, such as Polyimide or Polyvinylidenefluoride, used for Electro-Bonded Laminates (EBLs). The properties of the well known VHB 4910 acrylic elastomer are presented to illustrate how the permittivity and the leakage current can be measured as a function of the electric field and the deformation state, using the proposed set-up. The material properties were measured on membranes under different fixed pre-stretch conditions $\left(\lambda_{1}, \lambda_{2}=3,4,5\right)$, in order to eliminate effects due to the change in sample geometry, using gold sputtered electrodes, $20 \mathrm{~nm}$ thick. The values obtained for the permittivity of the material are in good agreement with the work of other authors. The dissipative properties revealed by the measurements performed at high fields, similar to the ones encountered in operation, indicate that this less investigated aspect of VHB needs to be taken in consideration for real world applications.
\end{abstract}

Keywords: Dielectric elastomer actuators, acrylic elastomers, dielectric properties, insulating properties

\section{INTRODUCTION}

Unlike materials such as piezoelectrics, DEAs and EBLs exploit electrostatic field in a direct way, namely as the Coulomb force between the charges that have been separated by applying a potential across the dielectric.

In the case of DEAs, the dielectric is highly compliant and deforms due to the Maxwell stress applied to it, as shown in figure 1a, thus transforms part of the energy stored in the electrostatic field into mechanical energy. The actuation behavior of DEAs is governed by the mechanical properties as well as the dielectric properties of the dielectric, whereas the stress is given by equation 1 and the consequent deformation is described by the mechanical constitutive equation of the material.

Further author information: (Send correspondence to Andrea Bergamini)

Andrea Bergamini: E-mail: andrea.bergamini@empa.ch, Telephone: 41587654424

Electroactive Polymer Actuators and Devices (EAPAD) 2011, edited by Yoseph Bar-Cohen, Federico Carpi, Proc. of SPIE Vol. 7976, 79763B - (c) 2011 SPIE · CCC code: 0277-786X/11/\$18 · doi: 10.1117/12.894558 
In the case of the EBLs, no actuation action is obtained by applying an electrostatic field across the dielectric. The ability of interface within the dielectric to transfer shear stress can be activated by exerting a normal stress across it while relying on the presence of friction, as shown in figure 1b. The change in bending (and torsional) stiffness in EBLs is based on the application of a strong electric field across a stiff dielectric that contains an interface. Shear stress transfer is enabled by normal stress combined with friction.
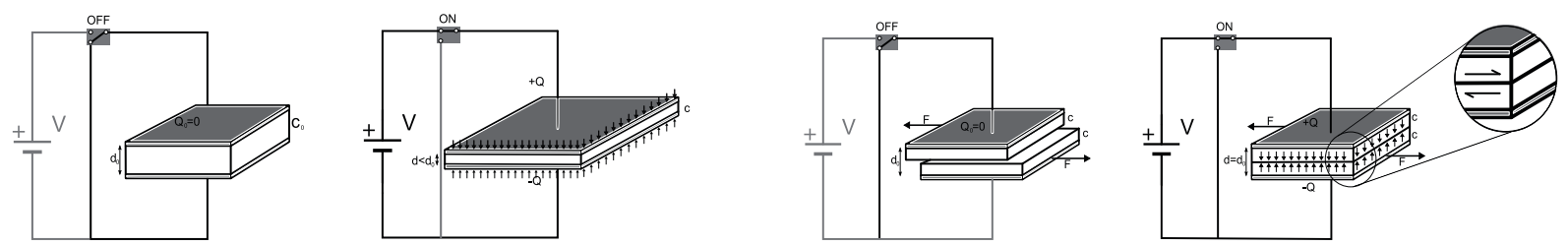

Figure 1. Left:(a) Schematic representation of the working principle of DEAs. The incompressible elastomer change thickness and consequently length and width under the effect of the applied Maxwell stress. Right:(b) The dielectric of EBLs is several order of magnitude stiffer than the one of DEAs, hence no substantial deformation is observed. In exchange, the shear stress transfer at the interface within the dielectric is modulated by the Maxwell stress.

In both cases, the mechanical characterization of the dielectric relies on well known procedures. The description of the non-linear behavior of DEA materials such as the $3 \mathrm{M}^{T M} \mathrm{VHB}^{T M} 49 \mathrm{XX}$ and the IPN derived from it has been object of several investigations. ${ }^{1,2}$

\section{PERMITTIVITY MEASUREMENT}

The permittivity measurements on polymeric dielectrics has also been object of the work of several groups. ${ }^{2-6}$ The permittivity of a dielectric material is customarily measured by analyzing the capacitive component in an LCR circuit by means of an impedance analyzer (LCR-meter). This was also the case in the above mentioned studies. The permittivity of the material within the electrodes can be determined with good accuracy, based on the geometry of the sample. Impedance analyzers are generally operated at low voltage (of the order of $0 \cdots 5 \mathrm{~V}$ ) and frequencies between 100 and $10{ }^{6} \mathrm{~Hz}$. The dielectric and dissipative properties of materials are calculated based on the measurement of the alternating current flowing in the tested object when an alternating voltage is applied to it, as sketched in figure 2

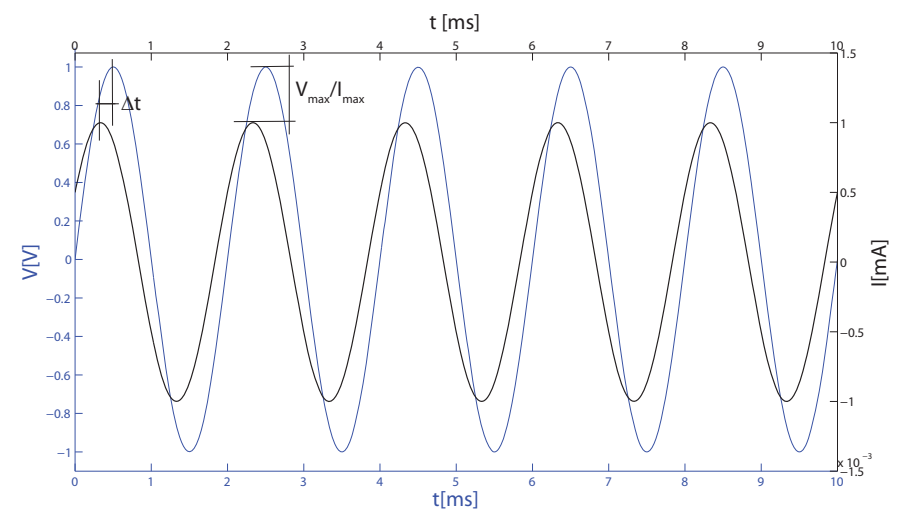

Figure 2. The permittivity and resistivity of materials is measured using LCR Meters, generally assuming a capacitor and resistor connected in parallel, based on the complex impedance of the circuit.

There are two drawbacks to the use of this method for the determination of the dielectric properties of polymer films:

1. The permittivity of polymers is strongly frequency dependent.

2. The dissipative behavior of polymers is field dependent. 
The frequency of operation as well as the field intensity at which DEAs and EBLs are activated, strongly differ from the conditions under which their dielectric and insulating properties are measured. Thus, the possibility is left open that under operational conditions the electrical behavior of DEA and EBL materials may be different than what is assumed based on conventional measurement methods. This has lead to the development of a set-up that allows for the investigation of the dielectric and insulating properties of thin polymer films.

\section{PROPOSED SET-UP}

As shown in figure 3, the charge current for a capacitor on which a linear voltage ramp is imposed is directly proportional to the permittivity of the dielectric. If all other parameters are known, the permittivity $\epsilon$ of the material can be easily calculated as:

$$
\epsilon=\frac{I \cdot d}{\epsilon_{0} \cdot A \cdot \frac{d V}{d t}}
$$

Where $I$ is the charging current, $d$ is the thickness of the dielectric sample, $\epsilon_{0}$ is the permittivity of vacuum, $A$ is the area of the sample and $\frac{d V}{d t}=\dot{\mathrm{V}}$ is the slope of the applied voltage ramp.

For a polymer sample with a thickness of $40 \mu \mathrm{m}$, a diameter of $20 \mathrm{~mm}$ and a permittivity $\epsilon=4$ the charge current for a voltage ramp $\dot{V}=200 \mathrm{~V} / \mathrm{s}$ is of the order of $6 \mathrm{nA}$.
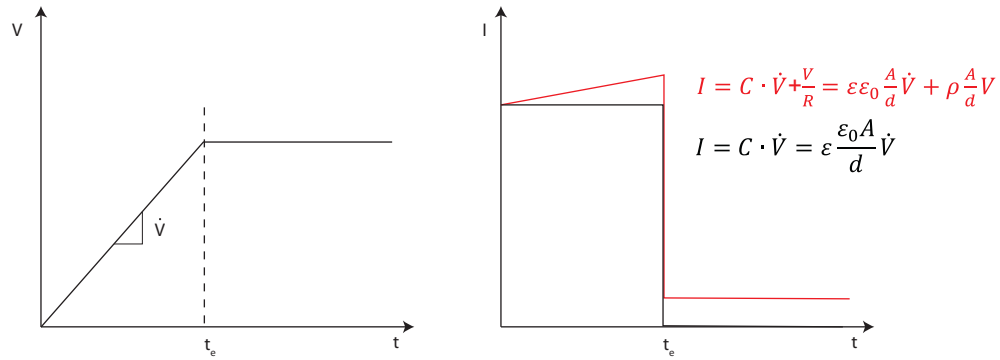

Figure 3. The charge current of and ideal (black line) and a real, i.e. in parallel with a resistor, capacitor (red line), is proportional to the permittivity of the dielectric.

Figure 4 shows the set-up for the the measurement of the charge current flowing through the polymer samples, as proposed by Di Lillo et al. 7,8 The ramp signal is generated by programming a suitable signal into the signal generator (Agilent 33120A Arbitrary Waveform generator). The signal is amplified by a high voltage amplifier (Trek 20/20C). The current flowing is converted to a voltage signal by a trans-impedance amplifier built in the laboratory and recorded by a oscilloscope (Tektronix TDS5034B).

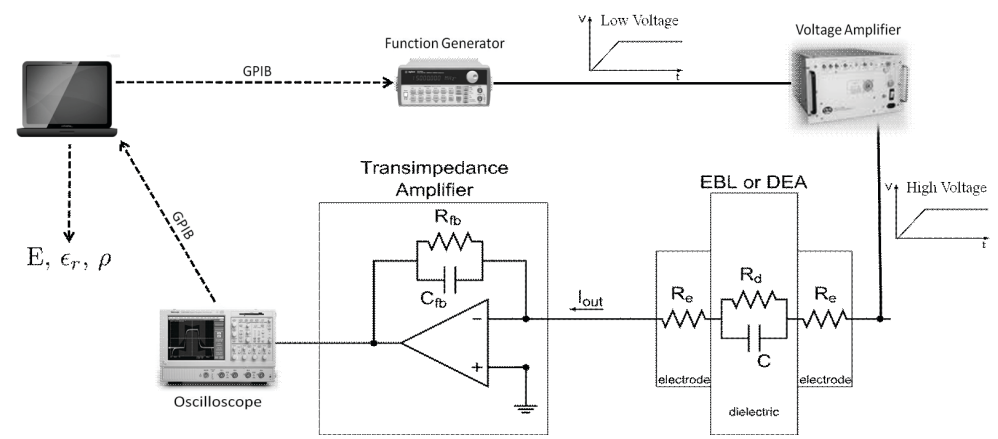

Figure 4. Set-up for the measurement of the charge current of small capacitors under a constant voltage ramp.

The charge current was measured for three different pre-stretch ratios $\lambda_{1}=\lambda_{2}=\lambda=3,4,5$. On each VHB sample stretched to $\lambda=3,4,5$, three pairs of electrodes (one on each face of the sample) where applied by sputtering, as visible 
in figure 5. Each area was named according to the color of the leads ('red', 'green', 'black'). The voltage ramp was kept constant at $200 \mathrm{~V} / \mathrm{s}$ for all measurements. Measurements were performed to different target voltages that decreased with increasing value of $\lambda$. The maximum target voltage $V_{\max }$ for $\lambda=3$ was $5000 \mathrm{~V}$, for $\lambda=4 V_{\max }$ was $4000 \mathrm{~V}$, for $\lambda=5$ $V_{\max }$ was $3000 \mathrm{~V}$. In order to suppress the deformation of the dielectric and the consequent failure of the sputtered gold electrodes, the samples were stiffened on both sides with common adhesive tape.

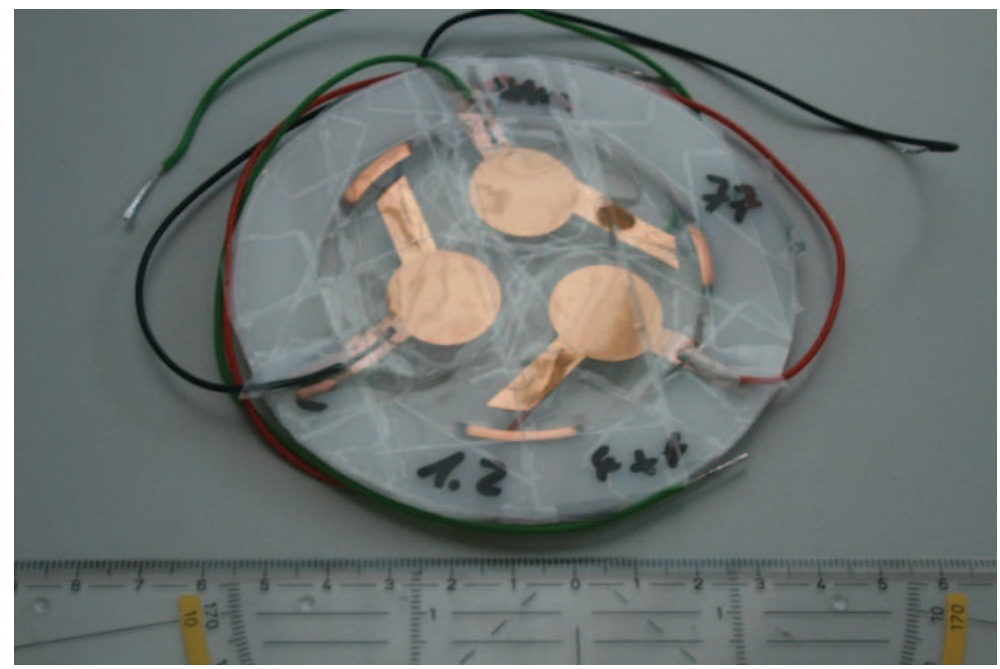

Figure 5. A VHB sample ready for measurement. Three measurement areas were applied on each VHB sample by sputtering gold electrodes on each face of the film.

\section{RESULTS AND DISCUSSION}

Figure 6 shows two typical examples of the recorded voltage and current values. While the behavior observed in figure 6a is approximately the one of an ideal capacitor (with only a very slight positive slope that corresponds to a resistor with an Ohmic characteristic), the current recorded for a film subjected to high field (figure 6b) clearly deviates from the behavior of the two situations depicted in figure 3. The current measured after the maximum voltage is reached and kept at that level, is considered to be the leakage current in the sample. Next to the obvious increase in the value of the current, a comparison of figures $6 \mathrm{a}$ and $6 \mathrm{~b}$ shows that at high fields the leakage current is also a function of time. These observations indicate a non-ohmic characteristic of the dissipative component of the sample at high fields.

For each measured area, 5 consecutive measurements were performed at intervals of a few minutes. It is noticeable how the first measurement (marked as '1st loop' in figure 6b) differs from the subsequent measurements. While for low target voltages all measurements were quite similar, a difference between the first and subsequent measurements could be observed at high target voltage and high stretches (i.e. for the highest fields).

Figure 7a shows the permittivity calculated based on the measured current and the geometry of the samples as a function of the applied field intensity $E$. The thickness of the samples used to calculate the permittivity $\epsilon$ and the field $E$ was assumed to be the initial thickness of the film (1mm for VHB 4910) divided by $\lambda^{2}$. The dissipative (leakage) component of the current measured at a fixed time after reaching the maxium voltage was subtracted from the measured current to obtain the charge current.

Figure $7 \mathrm{~b}$ shows the volume resistivity of the material as a function of $E$. The results displayed in the figure show that the volume resistivity of the investigated material increases very strongly (notice the logarithmic scale for the resistivity) for fields exceeding $40 \mathrm{MV} / \mathrm{m}$.

From the values measured for the permittivity and volume resistivity of the material it is possible to calculate values for the stored energy density (figure 8a) and dissipated power density (figure 8b). The calculated stored energy density increases quadratically with the field, as can be expected for a material with field-independent dielectric constant. The dissipated power appears to increase in a more than quadratic manner with respect to the applied field, for fields exceeding approximately $40 \mathrm{MV} / \mathrm{m}$. 

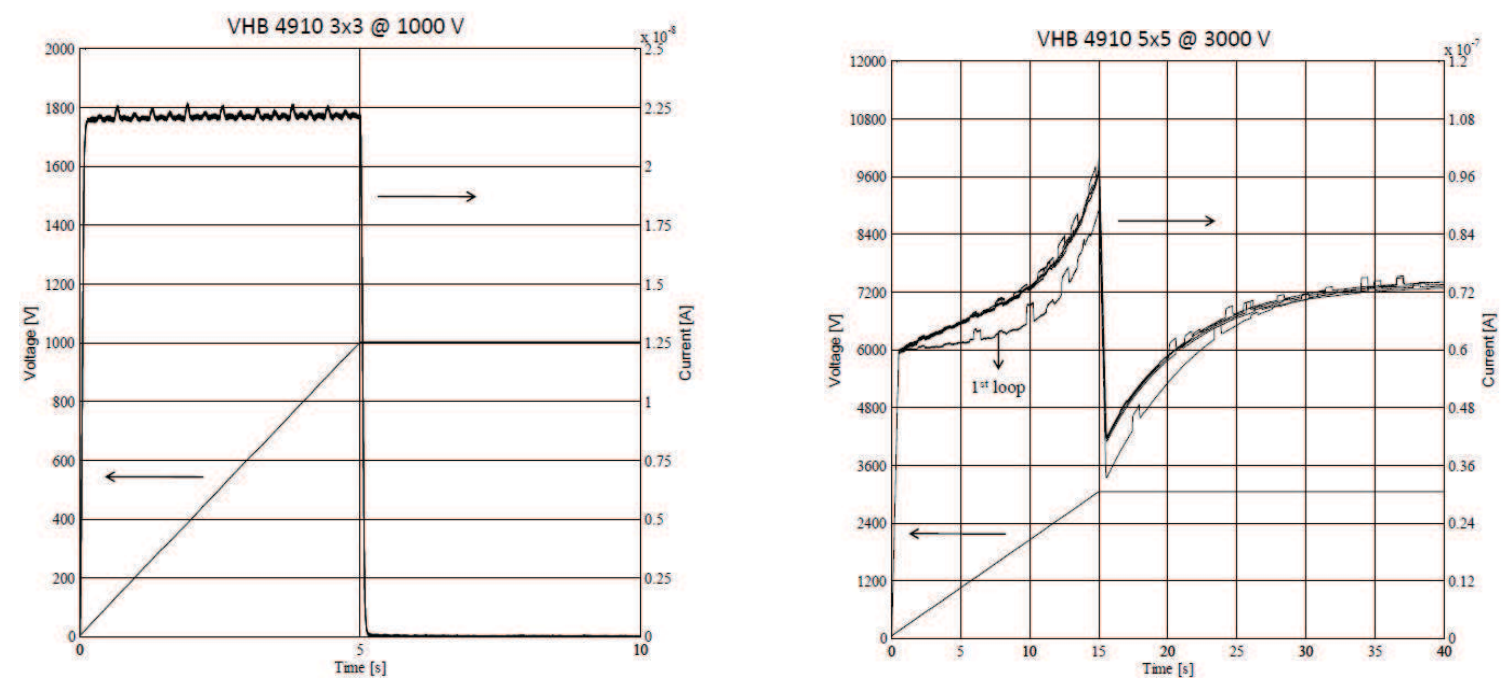

Figure 6. (a) voltage and current measured on a $\lambda=3$ sample with a target voltage of $1000 \mathrm{~V}$. (b) voltage and current measured on a $\lambda=3$ sample with a target voltage of $3000 \mathrm{~V}$
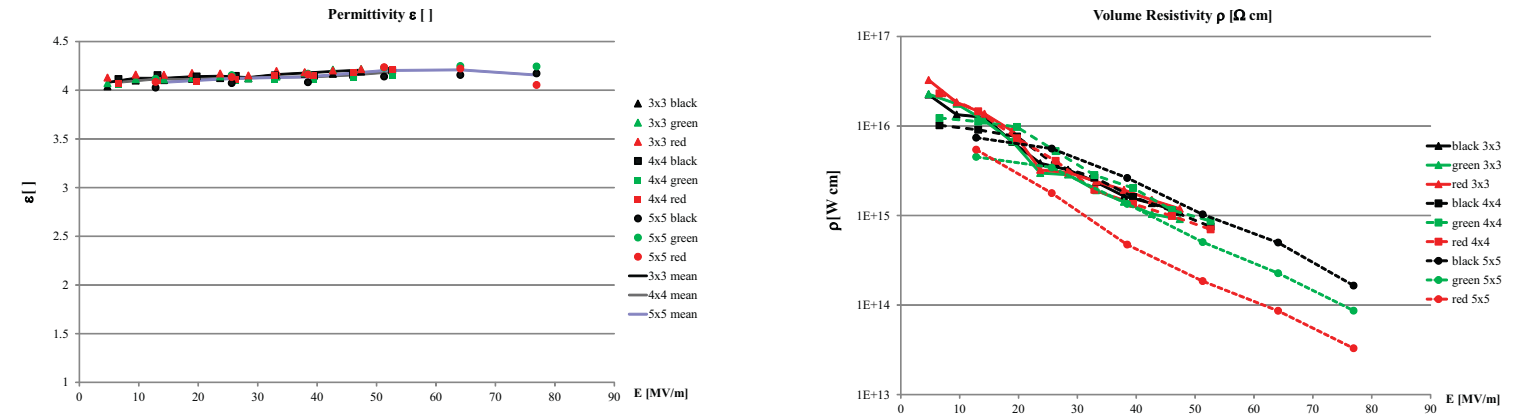

Figure 7. (a) Permittivity of VHB as a function of the applied field (defined as $V_{\text {max }} / d_{\text {nominal }}$, where $d_{n}$ ominal $=1 \mathrm{~mm} / \lambda^{2}$ ). The dissipative current component, due to the finite resistivity of the sample, was subtracted prior to calculating the permittivity according to (2). (b) Volume resistivity of the samples calculated at $V_{\max }$ as a function of the applied field.
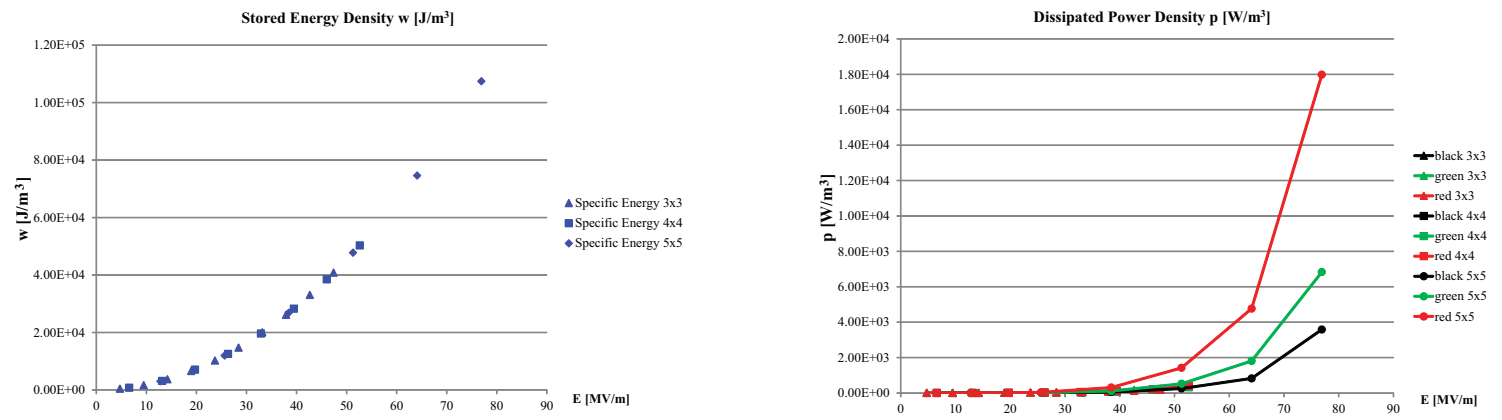

Figure 8. (a) Stored energy density in VHB 4910 as calculated based on an average permittivity value of 4.1 , as a function of the applied field $E$. (b) Dissipated power density calculated based on the applied voltage and measured volume resistivity, as a function of the applied field $E$.

\section{CONCLUSIONS AND OUTLOOK}

The set-up presented in this contribution allows for the measurement of the dielectric and insulating properties of thin polymer dielectrics, as they are found in EBLs and DEAs. Specifically, the permittivity and volume resistivity of VHB 4910 films at pre-stretch and field levels comparable to the ones found in real applications of this material for DEAs have been investigated. 
The results obtained for the dielectric constant are in the range of the values published by other groups. ${ }^{2-6}$ The dependance of the permittivity of VHB from the level of prestretch previously reported by our group could not be verified. This finding can rather be attributed to the fact that unlike in previous measurements, the electrodes were applied directly to the material by sputtering instead using solid electrodes, rather than to the use of the proposed measurement set-up. Initial measurements (not presented in this work) made with the same solid electrodes as used for previous measurements ${ }^{2}$ revealed an irregular charging current at high voltages and prompted for the use of sputtered gold electrodes. While it was not possible to conclusively demonstrate it, the assumption was made that the irregular charging current curves observed when using solid electrodes and the pre-stretch dependency of the dielectric constant of VHB previously reported may be attributed to the presence of air gaps between the electrodes and the VHB film. The use of sputtered electrodes eliminated both phenomena.

The measurement of the volume resistivity of VHB under high field conditions is considered to represent the most innovative contribution of the present work. The results shown in figure $7 \mathrm{~b}$ are in good agreement with the volume resistivity values reported by Molberg et. $\mathrm{al}^{4}$ at fairly low field levels. The values recorded for electric field intensities exceeding $40 \mathrm{MV} / \mathrm{m}$ show an aspect of the behavior of this material that have not been previously reported based on direct electrical measurements, while indirect evidence of the dissipative behavior is reported based on mechanical measurements was by Plante et al. ${ }^{9}$

A comparison of the specific stored energy and specific dissipated power with respect to the applied field, as shown in figures 8a and 8b shows clearly that, for the operation of VHB based DEAs, the dissipative behavior of the material cannot be neglected when making considerations about the efficiency of such systems. This is expected to be especially important for energy harvesting applications.

In the future, the dielectric and insulating properties of other candidate materials for DEA applications shall be investigated and compared to the ones of VHB. Also, the mechanisms leading to the non-ohmic behavior of VHB shall be investigated, especially in view of the development of materials with improved insulating properties. The measurement of the evolution of the volume resistivity as a function of the applied field is deemed interesting especially as an indicator of imminent electrical breakdown. More investigations shall be devoted to this point as well as to the implications that the thermal effects of the dissipation of electrical power may have on the durability of DEAs, especially in the case of devices with low specific surfaces, such as stacked actuators.

\section{ACKNOWLEDGMENTS}

This project was partly funded by the Swiss National Science Foundation, National Research Program 62, Smart Materials under Grant Agreement No. $406240 \_1261130$ and partly by the Swiss National Science Foundation under Grant Agreement No. 200021-107661. The authors wish to acknowledge the financial support they received for this work.

\section{REFERENCES}

[1] Schmidt, A. e. a., "Multiaxial mechanical characterization of interpenetrating polymer network reinforced acrylic elastomer," Experimental Mechanics In print, doi:10.1007/s11340-010-9462-z (2011).

[2] Wissler, M., MODELING DIELECTRIC ELASTOMER ACTUATORS, PhD thesis, ETH Zurich (2007).

[3] McKay, T. e. a., "The dielectric constant of 3m vhb: a parameter in dispute," in [Proc. of SPIE Vol 7287], (2009).

[4] Molberg, M. e. a., "Frequency dependent dielectric and mechanical behavior of elastomers for actuator applications," Journal of Applied Physics 106 (2009).

[5] Jean-Mistral, C. e. a., "Dielectric properties of polyacrylate thick films used in sensors and actuators," Smart Materials and Structures 19 (2010).

[6] Kofod, G. e. a., "Actuation response of polyacrylate dielectric elastomers," Journal of Intelligent Materials and Structures 14 (2003).

[7] Di Lillo, L. e. a., "Dielectric materials for variable stiffness skins in shape adaptation applications," in [Proceedings of the International Conference on Adaptive Structures and Technologies ], (2010).

[8] Di Lillo, L. e. a., "Quasi-static electric properties of insulating polymers at high voltage for electro bonded laminates (ebl),," Smart Materials and Structures Submitted (2011).

[9] Plante, J. e. a., "On the performance mechanisms of dielectric elastomer actuators," Sensors and Actuators A 137, 96-109 (2007). 